\title{
Lung cancer randomized controlled trials should compare stereotactic body radiation therapy with observation, NOT surgery
}

\author{
Raja M. Flores, MD
}

\author{
From the Department of Thoracic Surgery, Icahn School of Medicine at Mount Sinai, Mount Sinai Health System, \\ New York, NY. \\ Disclosures: Author has nothing to disclose with regard to commercial support. \\ Received for publication Aug 9, 2017; accepted for publication Aug 19, 2017; available ahead of print Sept 13, \\ 2017. \\ Address for reprints: Raja M. Flores, MD, Department of Thoracic Surgery, Mount Sinai Health System, Icahn \\ School of Medicine at Mount Sinai, One Gustave L. Levy Place, Box 1023, New York, NY 10029 (E-mail: \\ raja.flores@mountsinai.org). \\ J Thorac Cardiovasc Surg 2018;155:403-4 \\ $0022-5223 / \$ 36.00$ \\ Copyright $(C) 2017$ by The American Association for Thoracic Surgery \\ http://dx.doi.org/10.1016/j.jtcvs.2017.08.058
}

The debate between surgery and stereotactic body radiation therapy (SBRT) has devolved into a territorial battle of subspecialties. We are doctors before we are surgeons or radiation oncologists. We must focus on what is best for our patients. If SBRT one day proves to be better at curing lung cancer, then we will gladly put aside our scalpels and embrace the change. However, the preponderance of evidence-based data available today indicate that surgery is the only treatment modality resulting in a cure for stage I lung cancer.

The Veterans Affairs study by Cornwell and colleagues ${ }^{1}$ demonstrates better survival and lower recurrence rates with surgery than SBRT. Of course we are biased, so are radiation oncologists evaluating reports favoring SBRT. Does the hazy inflammatory reaction created by SBRT on computed tomography (CT) scan indicate cure or does this just mask the underlying malignancy? Because retrospective studies have their limitations, we look to randomized controlled trials (RCTs) to answer important questions like this one.

There is a false perception that equipoise exists despite 3 failed RCTs comparing surgery with SBRT. Most recently, a great deal of enthusiasm has been created for SBRT by a severely flawed study in The Lancet Oncology evaluating a combined analysis of 2 failed RCTs, inaccurately presented as level 1 evidence and accompanied by a sycophantic editorial commentary akin to placing "lipstick on a pig." "2,3 There is absolutely no evidence that SBRT is superior or even equivalent to surgery based on these data. There is zero level 1 evidence that SBRT cures lung cancer, despite the lipstick.

Many retrospective SBRT studies include subjects without histologically confirmed lung cancer, indolent lung cancers, lesions of unspecified size and location, loose definitions of local recurrence, and survival times at 1 and 3 years, not the standard 5-year survival mark. These studies do not take into account CT features of the primary lesion,

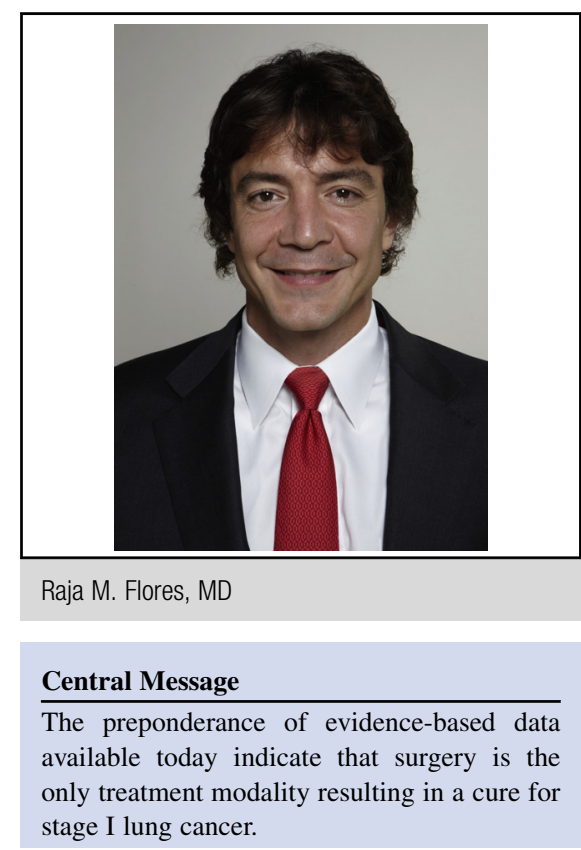

See Article page 395.

such as solid versus part-solid versus pure ground-glass opacities. These features significantly influence survival on the basis of biological factors alone. Pure ground-glass opacities and part-solid features on CT scan suggest indolent lung cancers that can demonstrate excellent 5-year survivals with observation alone.

The question should not be "Is SBRT better than surgery?" It should be "Is SBRT better than observation?" We already have an RCT that unequivocally proves the superiority of surgery to observation: the National Lung Screening Trial. ${ }^{4}$ Until we have a trial that proves SBRT is better than observation, we are committing academic medical malpractice by embarking on another surgical RCT. As doctors and investigators, we must not subject healthy curable individuals to an unproven therapy under the guise of an RCT. The only logical next step is to first prove whether or not SBRT is better than observation. Only then can we advance to the next step of comparing SBRT with surgery in healthy patients.

\section{References}

1. Cornwell LD, Echeverria AE, Samuelian J, Mayor J, Casal RF, Bakaeen FG, et al Video-assisted thoracoscopic lobectomy is associated with greater recurrence-free 
survival than stereotactic body radiotherapy for clinical stage I lung cancer. $J$ Thorac Cardiovasc Surg. 2018;155:395-402.

2. Chang JY, Senan S, Paul MA, Mehran RJ, Louie AV, Balter P, et al. Stereotactic ablative radiotherapy versus lobectomy for operable stage I non-small-cell lung cancer: a pooled analysis of two randomised trials. Lancet Oncol. 2015;16: 630-7. Erratum in: Lancet Oncol. 2015;16:e427.
3. Treasure T, Rintoul RC, Macbeth F. SABR in early operable lung cancer: time for evidence. Lancet Oncol. 2015;16:597-8.

4. Aberle DR, DeMello S, Berg CD, Black WC, Brewer B, Church TR, et al; National Lung Screening Trial Research Team. Results of the two incidence screenings in the National Lung Screening Trial. N Engl J Med. 2013;369: 920-31. 